Gut and Liver, Vol. 9, No. 4, July 2015, pp. 540-546

\title{
Intraductal Ultrasonography without Radiocontrast Cholangiogram in Patients with Extrahepatic Biliary Disease
}

\author{
Sung-Uk Lim*, Chang-Hwan Park*, Won-Ju Kee*, Jeong-Hyun Lee*, Soo-Jung Rew ${ }^{\dagger}$, Seon-Young Park*, Hyun-Soo Kim*, \\ Sung-Kyu Choi*, and Jong-Sun Rew* \\ *Division of Gastroenterology, Department of Internal Medicine, Chonnam National University Medical School, and ${ }^{\dagger}$ Department of Internal \\ Medicine, Gwangju Christian Hospital, Gwangju, Korea
}

Background/Aims: Intraductal ultrasonography (IDUS) has been performed as an adjunct to endoscopic retrograde cholangiography (ERC) during radiocontrast cholangiography $(\mathrm{RC})$. Radiation exposure during RC poses a health risk to both patients and examiners. We evaluated the feasibility of IDUS without RC in various extrahepatic biliary diseases. Methods: IDUS was performed with the insertion of an IDUS probe from the papilla of Vater to the confluent portion of the common hepatic duct without fluoroscopy. The technical success rate and procedure-related complications were evaluated retrospectively. Results: Wire-guided IDUS without RC was performed in 105 patients. The mean age was 66.5 years, and 50 (47.6\%) were male. The IDUS diagnoses included choledocholithiasis $(73,69.5 \%)$, benign biliary stricture (11, $10.5 \%)$, choledocholithiasis with biliary pancreatitis $(9,8.6 \%)$, bile duct cancer $(5,4.8 \%)$, pancreatic cancer $(1,0.9 \%)$, and others (6, 5.7\%). After IDUS, 66 (62.8\%) underwent stone removal, 19 (18.1\%) underwent biliary drainage, and 7 (6.6\%) underwent brush cytology and biopsy. No significant complications such as perforation or severe pancreatitis occurred. Conclusions: IDUS without RC was a feasible and safe approach in patients with various extrahepatic biliary diseases. We anticipate a potentially important role of IDUS in various ERC procedures because it lacks the hazards of RC. (Gut Liver 2015;9:540-546)

Key Words: Intraductal ultrasonography; Radiocontrast cholangiography; Endoscopic retrograde cholangiography

\section{INTRODUCTION}

Standard endoscopic retrograde cholangiography (ERC) is performed under radiographic visualization of the biliary tree after the bile duct is filled with iodinated contrast agent. During radiocontrast cholangiogram, the radiation used poses a health threat to both examiners and patients. Radiocontrast cholangiogram is a hardship in special clinical situations such as the examination of pregnant women and critically ill patients in an intensive care unit. ${ }^{1-4}$ The contrast medium used for the cholangiogram could also provoke a hypersensitivity reaction in sensitized patients. ${ }^{5}$ Therefore, endoscopic retrograde ultrasonic cholangiogram without radiocontrast cholangiogram, minimizing the radiation exposure, would be desirable for both examiners and patients. However, it is still controversial which imaging method would be suitable for the replacement of fluoroscopy for ERC.

Intraductal ultrasonography (IDUS) allows real-time crosssectional imaging of the biliary tract, and is one of the most useful diagnostic tools for various extrahepatic biliary diseases. ${ }^{6-8}$ IDUS has been performed as an adjunct to ERC under radiocontrast cholangiogram. If it is practicable to perform IDUS without radiocontrast cholangiogram, it could be used as a fundamental imaging method, replacing the radiocontrast cholangiogram for ERC. The aim of this study was to evaluate the feasibility and safety of implementing IDUS without radiocontrast cholangiogram in patients with various extrahepatic biliary diseases.

\section{MATERIALS AND METHODS}

\section{Patients}

The clinical records of 105 patients who underwent IDUS without radiocontrast cholangiogram from November 2013 to December 2013 at Chonnam National University Hospital were

\footnotetext{
Correspondence to: Chang-Hwan Park

Division of Gastroenterology, Department of Internal Medicine, Chonnam National University Medical School, 160 Baekseo-ro, Dong-gu, Gwangju 501-746, Korea

Tel: +82-62-220-6977, Fax: +82-62-225-8578, E-mail: p1052ccy@hanmail.net

Received on May 27, 2014. Revised on September 18, 2014. Accepted on October 6, 2014. Published online May 13, 2015 pISSN 1976-2283 eISSN 2005-1212 http://dx.doi.org/10.5009/gnl14200

@ This is an Open Access article distributed under the terms of the Creative Commons Attribution Non-Commercial License (http://creativecommons.org/licenses/by-nc/4.0) which permits unrestricted non-commercial use, distribution, and reproduction in any medium, provided the original work is properly cited.
} 
analyzed. The inclusion criteria were choledocholithiasis, biliary pancreatitis, indeterminate biliary stricture, and periampullary tumor. This retrospective study was performed in accordance with the guidelines of the Institutional Review Board (IRB number: CNUH-2014-011).

\section{Methods}

All procedures were performed using a standard side-viewing duodenoscope (JF-260F or TJF-160F; Olympus, Tokyo, Japan) in an endoscopy room without radiocontrast cholangiogram. After cannulation with a 0.035-inch-diameter guidewire (Jagwire; Boston Scientific, Natick, MA, USA), the "bile aspiration" technique or checking of the guidewire direction on fluoroscopy were used to indicate bile duct cannulation. ${ }^{3}$ Some patients in who there was difficultly accessing the bile duct underwent infundibulotomy or a precutting method on the ampulla using a needle knife (Microknife XL; Boston Scientific). A 2.0-mmdiameter IDUS probe with a frequency of 20-MHz (UM-G20-29R; Olympus) was advanced over a guidewire into the bile duct. IDUS scanning was performed while inserting the IDUS probe from the papilla of Vater (POV) to the confluent portion of the common bile duct (CBD) over the guidewire. Stones, biliary stricture, and in-growing or out-growing masses were revealed by IDUS (Fig. 1).

\section{Definitions}

Technical success was defined as successful placement of the IDUS probe into the confluent portion of the common hepatic duct. The procedure time required for IDUS was defined as the duration from when the duodenoscope visualized the POV to when the IDUS probe was extracted from the papillary opening. Procedural-related complications were defined as any kind of newly developed complications after the IDUS procedure, such as bleeding, perforation, pancreatitis, and abdominal pain. PostIDUS pancreatitis was defined as the presence of abdominal pain with post-IDUS elevation of amylase. Post-IDUS amylase elevation was defined as the elevation of serum amylase by more than 3 times the normal range and basal level. ${ }^{9}$

\section{Statistical analysis}

All statistical analyses were performed using SPSS software version 20.0 (SPSS Inc., Chicago, IL, USA). Categorical variables were described using frequencies and percentages. Continuous variables were summarized using the mean and standard deviation. Comparisons between groups were performed using the Mann-Whitney U-test, the Wilcoxon U-test, and the chi-square
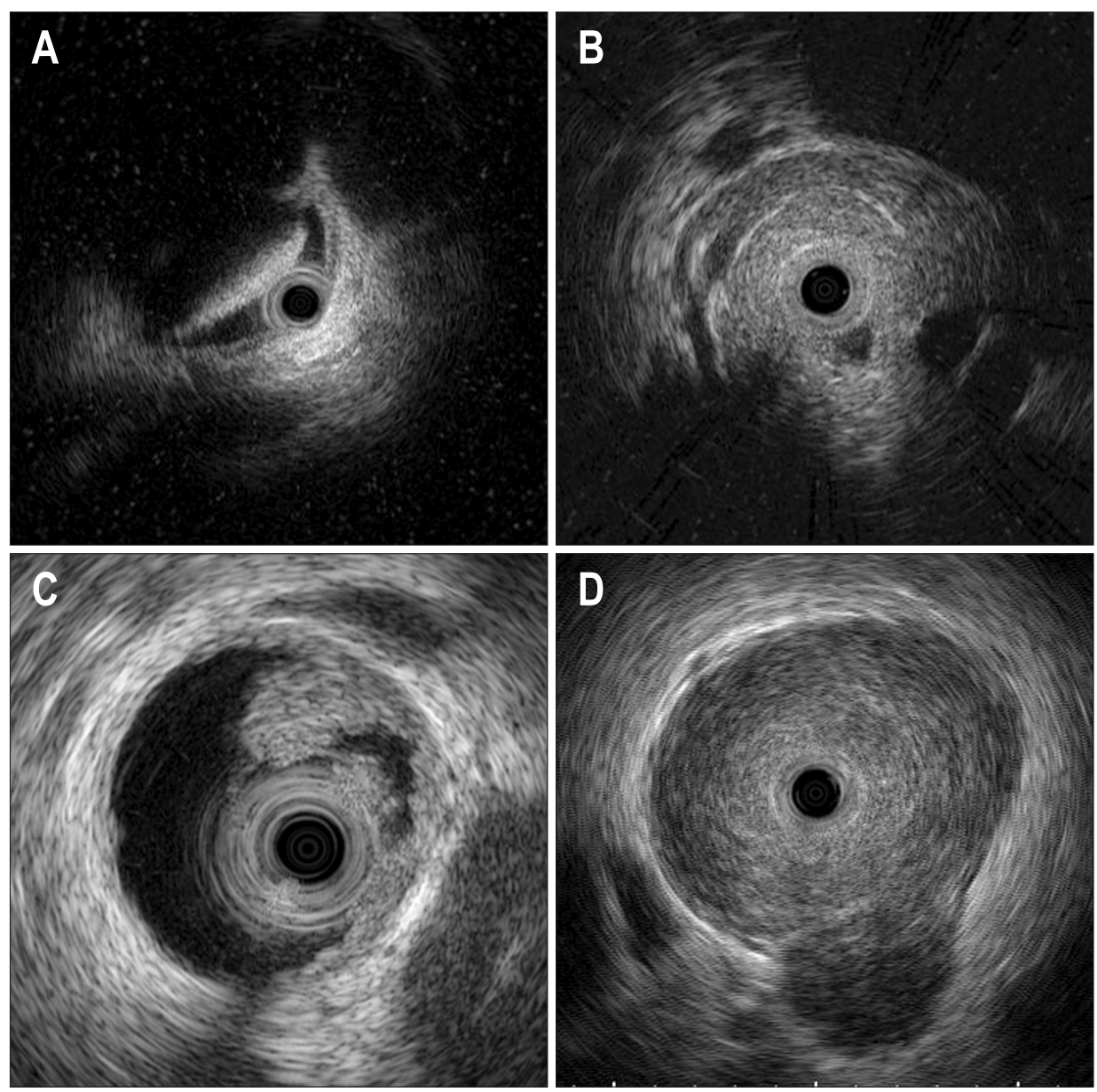

Fig. 1. Intraductal ultrasonographic findings. (A) Stone in the common bile duct. (B) Benign stricture. (C) Cholangiocarcinoma in situ. (D) Fungating cholangiocarcinoma. 
test, as appropriate. p-values $<0.05$ were considered significant.

\section{RESULTS}

\section{Patient characteristics}

A total of 105 patients underwent wire-guided IDUS without radiocontrast cholangiogram (Table 1). The mean age of the patients was 66.5 years, and 50 of the patients (47.6\%) were men. Two of the patients had a history of anaphylaxis to the iodinebased contrast agent. Forty-one patients (39.0\%) were clinically diagnosed with recurrent choledocholithiasis and had a history of previous endoscopic sphincterotomy (EST). Periampullary diverticulum (PAD) was noted in 31 patients (29.5\%), with the following subgroups: Type I, ampullary orifice in the diverticulum (8, 7.6\%); Type II, ampullary orifice in the margin of the diverticulum (9, 8.6\%); and Type III, ampullary orifice beyond the diverticulum $(14,13.3 \%)$.

\section{IDUS findings and outcomes}

Wire-guided IDUS without radiocontrast cholangiogram was successfully performed in all patients (Table 2). Because of the difficulty with biliary cannulation, the precut technique was carried out in $13(12.4 \%)$ patients (nine patients, infundibulotomy; four patients, precut sphincterotomy). The mean diameter of CBD was $13.1 \pm 4.5 \mathrm{~mm}$, and the mean size of stones detected on IDUS was $6.4 \pm 3.5 \mathrm{~mm}$. Pneumobilia was detected in 24 cases (22.9\%). The mean procedure time required for IDUS was $8.2 \pm 4.0$ minutes. Fluoroscopy was used in 10 cases (9.5\%) for selective biliary cannulation, nine cases (8.5\%) for stone capture with a basket, four cases (3.8\%) for endoscopic retrograde biliary drainage (ERBD) insertion, and eight cases (7.6\%) for the clearance of remnant stones. The total mean fluoroscopy time was 86 seconds (range, 8 to 273 seconds). The diagnoses made through IDUS were choledocholithiasis $(73,69.5 \%)$, biliary stricture $(11$,

Table 1. Characteristics of Patients Undergoing Wire-Guided Intraductal Ultrasonography without Radiocontrast Cholangiogram

\begin{tabular}{lc}
\multicolumn{1}{c}{ Characteristic } & Value \\
\hline No. of patients & 105 \\
Age, yr & $66.5(27-90)$ \\
Gender, male:female & $50: 55$ \\
Anaphylaxis history to contrast agent & 2 \\
Periampullary diverticulum & $31(29.5)$ \\
Type I & $8(7.6)$ \\
Type II & $9(8.6)$ \\
Type III & $14(13.3)$ \\
Papilla state before cannulation & \\
Intact papilla & $64(61.0)$ \\
Previous sphincterotomy & $41(39.0)$ \\
\hline
\end{tabular}

Data are presented as number (\%) or mean (range).
10.5\%), choledocholithiasis for biliary pancreatitis (9, 8.6\%), bile duct cancer $(5,4.8 \%)$, pancreatic cancer $(1,0.9 \%)$, and others (6, 5.7\%; two bile duct dilatations due to extrinsic compression, four ampullary masses or enlargement for ampullary adenoma or carcinoma). Magnetic resonance cholangiopancreatography was performed in nine patients $(9 / 105,8.6 \%)$ before ERC for evaluation of suspicious malignancy. Computed tomography coronal reconstruction was very useful for longitudinal imaging of the biliary tree like in magnetic resonance cholangiopancreatography or ERC, and was performed before ERC in 89 patients (84.8\%). After IDUS, 66 of the patients (66/105, 62.8\%) underwent stone removal, 19 (19/105, 18.1\%) underwent biliary drainage, and 7 (7/105, 6.6\%) underwent brush cytology and

Table 2. Outcomes Following Wire-Guided Intraductal Ultrasonography without Radiocontrast Cholangiogram

\begin{tabular}{|c|c|}
\hline Outcome & Value \\
\hline \multicolumn{2}{|l|}{ Diagnoses of IDUS } \\
\hline Choledocholithiasis & $73(69.5)$ \\
\hline Biliary stricture & $11(10.5)$ \\
\hline Choledocholithiasis for biliary pancreatitis & $9(8.6)$ \\
\hline Cholangiocarcinoma & $5(4.8)$ \\
\hline Pancreatic cancer & $1(0.9)$ \\
\hline Others* & $6(5.7)$ \\
\hline Diameter of CBD, mm & $13.1 \pm 4.5$ \\
\hline Size of bile duct stone, mm & $6.4 \pm 3.5$ \\
\hline Pneumobilia on IDUS & $24(22.9)$ \\
\hline Procedure time, $\min$ & $8.2 \pm 4.0$ \\
\hline Fluoroscopic time, sec & $86.1 \pm 82.3$ \\
\hline Technical success & $105(100.0)$ \\
\hline \multicolumn{2}{|l|}{ Sphincterotomy for cannulation } \\
\hline None & 92 (87.6) \\
\hline Infundibulotomy & $9(8.6)$ \\
\hline Precut & $4(3.8)$ \\
\hline \multicolumn{2}{|l|}{ Subsquent procedure } \\
\hline Stone removal & $66(62.8)$ \\
\hline Biliary drainage & $18(17.1)$ \\
\hline Brush cytology and biopsy & $7(6.6)$ \\
\hline None & $14(13.3)$ \\
\hline ERC during following procedure & $17(16.2)$ \\
\hline \multicolumn{2}{|l|}{ Procedure-related complication } \\
\hline Bleeding & 0 \\
\hline Perforation & 0 \\
\hline Acute pancreatitis & $1(1.0)$ \\
\hline Abdominal pain & 0 \\
\hline
\end{tabular}

Data are presented as number (\%) or mean \pm SD.

IDUS, intraductal ultrasonography; CBD, common bile duct; ERC, endoscopic retrograde cholangiography.

*Others: dilatation due to extrinsic compression, ampullary mass or enlargement for ampullary adenoma or carcinoma. 
biopsy. The procedural-related complications observed included mild post-IDUS pancreatitis in one patient (1.0\%), following ERBD. No significant complications such as bleeding, perforation, or severe pancreatitis occurred.

\section{Comparisons of clinical characteristics and outcomes of IDUS along with papillary conditions before cannulation}

The clinical characteristics of the group with previous EST and the group with intact papilla are summarized in Table 3. No significant differences were seen between the two groups in terms of age, gender, IDUS diagnoses, presence of PAD, and therapeutic ERC procedures used. The technical success rate of IDUS was 100\% in both groups. The previous EST group did not require the precut technique or EST extension for selective biliary cannulation, as opposed to the intact papilla group (0\% vs $20.3 \%, p<0.001)$. However, pneumobilia was significantly higher in the previous EST group (20/41, 48.8\%) than in the intact papilla group $(4 / 64,6.3 \%)$. In addition, pneumobilia occurred often in patients with recurrent cholelithiasis (17/35, 48.6\%). However, diagnostic disturbance of pneumobilia was very rare $(1 / 24,4.1 \%)$. The procedure time required for IDUS was significantly shorter in the previous EST group than in the intact papilla group $(7.0 \pm 3.6$ minutes vs $8.9 \pm 4.0$ minutes, $p=0.018)$. No significant differences in IDUS-associated complications were observed between the groups.

Table 3. Comparisons with Papillary Condition before Cannulation

\begin{tabular}{|c|c|c|c|}
\hline & Previous EST $(\mathrm{n}=41)$ & Intact papilla $(\mathrm{n}=64)$ & p-value \\
\hline Age, mean (range), yr & $68.9(27-87)$ & $65.0(27-90)$ & 0.153 \\
\hline Gender, male:female & $18: 23$ & $32: 32$ & 0.529 \\
\hline Diagnoses of IDUS & & & 0.121 \\
\hline Choledocholithiasis & $32(78.1)$ & $41(64.1)$ & \\
\hline Biliary stricture & $4(9.8)$ & $7(10.9)$ & \\
\hline Choledocholithiasis for biliary pancreatitis & $3(7.3)$ & $6(9.4)$ & \\
\hline Cholangiocarcinoma & $1(2.4)$ & $4(6.3)$ & \\
\hline Pancreatic cancer & 0 & $1(1.6)$ & \\
\hline Others & $1(2.4)$ & $5(8.0)$ & \\
\hline MRCP before ERCP & $1(2.4)$ & $8(12.5)$ & 0.158 \\
\hline Periampullary diverticulum & $14(34.1)$ & $17(26.6)$ & 0.080 \\
\hline Type I & $5(12.2)$ & $3(4.7)$ & \\
\hline Type II & $6(14.6)$ & $3(4.7)$ & \\
\hline Type III & $3(7.3)$ & $11(17.2)$ & \\
\hline Pneumobilia on IDUS & $20(48.8)$ & $4(6.3)$ & $<0.001$ \\
\hline Technical success & $41(100)$ & $64(100)$ & 1.000 \\
\hline Precut technique & 0 & $13(20.3)$ & $<0.001$ \\
\hline Following ERC procedure & & & 0.108 \\
\hline Stone removal & 32 (78.0) & $35(54.7)$ & \\
\hline Biliary drainage & $4(9.8)$ & $12(18.7)$ & \\
\hline Brush cytology and biopsy & $2(4.9)$ & $6(9.4)$ & \\
\hline None & $3(7.3)$ & $11(17.2)$ & \\
\hline Procedure time, min & $7.0 \pm 3.6$ & $8.9 \pm 4.0$ & 0.018 \\
\hline Fluoroscopy time, sec & $110.2 \pm 79.8$ & $76.9 \pm 84.5$ & 0.459 \\
\hline Complications & & & 1.000 \\
\hline Mild pancreatitis & 0 & $1(1.6)$ & \\
\hline Bleeding & 0 & 0 & \\
\hline Perforation & 0 & 0 & \\
\hline Death & 0 & 0 & \\
\hline
\end{tabular}

Data are presented as number (\%) or mean \pm SD.

EST, endoscopic sphincterotomy; IDUS, intraductal ultrasonography; MRCP, magnetic resonance cholangiopancreatography; ERCP, endoscopic retrograde cholangiopancreatography; ERC, endoscopic retrograde cholangiography. 


\section{DISCUSSION}

In previous studies, IDUS was performed with radiocontrast cholangiogram as an adjunct to ERC. ${ }^{7,10,11}$ In the present study, it was performed without radiocontrast cholangiogram in a relatively large number of patients with various extrahepatic biliary diseases. After IDUS, 91 of the patients (86.6\%) underwent CBD stone removal, biliary drainage procedure, or brush cytology with biopsy. No significant complications such as bleeding, perforation, or severe pancreatitis were observed.

Radiation exposure during radiocontrast cholangiogram is harmful to both patients and examiners. Previous reports have demonstrated a linear relationship between the radiation dose and fluoroscopy time. ${ }^{11}$ In particular, radiation exposure during radiocontrast cholangiogram carries a potential risk to fetuses. The necessity for a nonradiation ERC technique during pregnancy has been presented in a number of studies. ${ }^{1-3}$ Many examiners are also exposed to the radiation from fluoroscopy. Although the use of lead protectors minimizes the risk of radiation exposure and the dose of a single ERC procedure is insufficient to cause harm, the accumulated dose of radiation should not be ignored in large-volume interventional endoscopists. ${ }^{12,13}$ In addition, the radiocontrast media can be harmful to patients with adverse reactions to iodine-containing contrast media. Iodine contrast media are detectable in the bloodstream after ERC, and systemic adverse reactions have been described. ${ }^{5}$ Radiocontrast cholangiogram is also not able to be performed on critically ill intensive care unit patients in need of ERC, because moving the patients to a critical-care setting with facilities for fluoroscopy presents a formidable obstacle. ${ }^{14}$ Therefore, performing ERC without radiocontrast cholangiogram is desirable for both examiners and patients.

It is controversial as to which imaging method could be used as a suitable replacement for the radiocontrast cholangiogram in ERC. It would be possible to use different imaging methods. First, contrast-enhanced ultrasonography (US) could replace the fluoroscopy for ERC. A case of contrast-enhanced US-guided ERC was reported for the treatment of CBD stones during pregnancy. ${ }^{15}$ However, the procedure requires further evaluation because experience with it has been limited. Second, endoscopic ultrasonography (EUS) could replace the radiocontrast cholangiogram for ERC. Artifon et al. ${ }^{16}$ reported EUS-guided sphincterotomy followed by stone extraction under US guidance to be feasible as a single-step intervention. Additional studies are required to validate their preliminary results. It is conceivable that a dual endoscope allowing both EUS imaging and therapeutic intervention will be developed to make the diagnosis and treatment of $\mathrm{CBD}$ stones with the same endoscope in the same setting feasible. ${ }^{16}$ Third, direct retrograde cholangioscopy (DRC) could also be used to replace the radiocontrast cholangiogram for ERC. DRC has been increasingly applied, thereby allowing high resolution video imaging and optical enhancement tech- niques. ${ }^{17}$ It was a useful complementary investigation to ERC in a selected group of patients with biliary disease. ${ }^{18}$ Future design developments of DRC will enable direct and easy access into the bile duct without any accessories in the near future. ${ }^{19}$ Fourth, IDUS could be applied to replace the radiocontrast cholangiogram for ERC. A recent study revealed IDUS to be a more accurate diagnostic modality for extrahepatic biliary diseases than any other examination. ${ }^{7}$ It has many benefits for ERC. First, it can be performed quickly and conveniently in most patients. ${ }^{6,20}$ Second, no special accessories or agents without a guidewire are needed for IDUS. However, contrast-enhanced US requires a contrast agent, DRC requires an anchoring balloon catheter for scope replacement, and EUS needs a linear array echoendoscope. Third, therapeutic ERC can be performed as a singlestep intervention under IDUS guidance.

In the present study, IDUS was performed easily without radiocontrast cholangiogram patients who had previously undergone EST. Neither the precut technique nor EST extension was needed in the previous EST group, causing the time required for IDUS to be significantly shorter than in the intact papilla group. In accordance with the IDUS findings, therapeutic ERC was performed as a single-step intervention. In addition, no significant IDUS-associated complications were observed in the previous EST group. These results suggest that IDUS could be considered as a first step diagnostic imaging modality for the evaluation of extrahepatic biliary disease in patients who have previously undergone EST.

Therapeutic ERC with IDUS was performed successfully without radiocontrast cholangiogram in most patients, except for 18 $(18 / 105,17.1 \%)$. These cases included therapeutic procedures involving difficult stone capture (nine patients), confirmation of ERBD location (four patients), difficult biliary recannulation after EST (one patient), and confirmation of stone clearance (four patients). Obviously, stone capture is easy with the assistance of radiocontrast cholangiogram. However, blind capture was possible in this study after checking the positions and sizes of stones with IDUS. If the blind capture method was unsuccessful 2 or 3 times, the location of the stone was then confirmed by fluoroscopy and captured with a fluoroscopy-aided basket without the use of radiocontrast cholangiogram. Radiocontrast cholangiogram was needed only in nine of the patients $(9 / 66$, 13.6\%) while removing CBD stones. The successful completion of the therapeutic procedure was confirmed with IDUS or radiocontrast cholangiogram. Stone clearance was confirmed by IDUS with basket sweeping 2 times ( 57 patients) or by radiocontrast cholangiogram (24 patients), and ERBD location was confirmed by bile aspiration and IDUS for guidewire positioning (eight patients) or radiocontrast cholangiogram (two patients) through all sessions (Fig. 2).

Pneumobilia was an important obstacle in the IDUS-assisted ERC procedure without radiocontrast cholangiogram. Pneumobilia was noted in 20 patients $(20 / 41,48.8 \%)$ who previously 


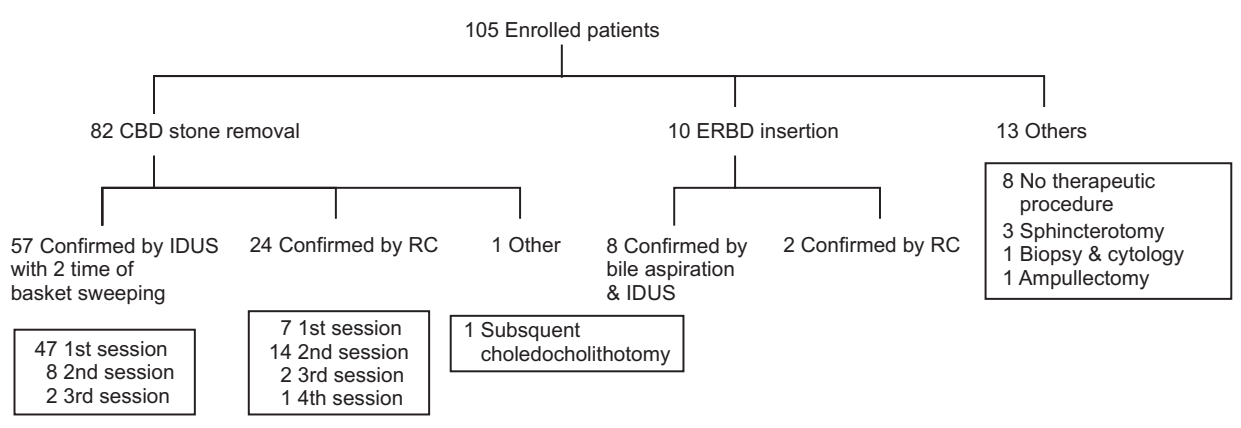

Fig. 2. Flow of therapeutic procedures through all endoscopic retrograde cholangiopancreatography sessions.

CBD, common bile duct; ERBD, endoscopic retrograde biliary drainage; IDUS, intraductal ultrasonography; $\mathrm{RC}$, radiocontrast cholangiogram. underwent EST. However, it was not difficult to differentiate between stones and air bubbles with saline flushing. First, in spite of obvious pneumobilia, small amounts of air did not disturb detection of the CBD stones. Air bubbles tended to be located on the upper biliary tree in the longitudinal section. While the position of the air bubbles was in the upper part, and stone position was lower part mostly on cross-sectional IDUS image. Second, echo finding of air reveals reverberation of fan-shaped and step ladder pattern, but that of stone shows hyper-acoustic crescent-like edge in probe side and posterior acoustic shadow in opposite side. Ang et al. ${ }^{21}$ revealed that water infusion method following IDUS after stone removal was more effective than radiocontrast cholangiogram and that stone and air bubble on cross section image of IDUS could be easily discriminated .

Recent studies have reported that mild pancreatitis occurs in $2 \%$ to $5 \%$ of patients as an IDUS-related complication. ${ }^{7}$ Those authors performed IDUS with a radiocontrast cholangiogram during or after ERC. ${ }^{22,23}$ In the present study, IDUS was performed without the radiocontrast cholangiogram. The only IDUS-associated complication was mild pancreatitis in one patient after ERBD (1.0\%). Filling the CBD with iodine-contrast media for conventional IDUS might increase the risk of pancreatitis. However, IDUS without the radiocontrast cholangiogram reduces the risk of pancreatitis by minimizing contrast injection into the pancreatic duct and by limiting papillary trauma. ${ }^{24}$ In addition, no significant complications related to inserting the IDUS probe were observed in the present study. These findings suggest that IDUS without the radiocontrast cholangiogram might be a safe procedure.

The present study had some limitations. First, the study was a nonrandomized retrospective single center study without a comparison group. Second, IDUS without fluoroscopy was performed by a single endoscopist (C.H.P.).

In conclusion, IDUS without the radiocontrast cholangiogram was feasible and safe in patients with various extrahepatic biliary diseases. We anticipate a potentially important role of IDUS in the field of various endoscopic retrograde cholangiopancreatography procedures without hazards of radiocontrast cholangiogram.

\section{CONFLICTS OF INTEREST}

No potential conflict of interest relevant to this article was reported.

\section{REFERENCES}

1. Shelton J, Linder JD, Rivera-Alsina ME, Tarnasky PR. Commitment, confirmation, and clearance: new techniques for nonradiation ERCP during pregnancy (with videos). Gastrointest Endosc 2008;67:364-368.

2. Akcakaya A, Ozkan OV, Okan I, Kocaman O, Sahin M. Endoscopic retrograde cholangiopancreatography during pregnancy without radiation. World J Gastroenterol 2009;15:3649-3652.

3. Rahmin MG, Hitscherich R, Jacobson IM. ERCP for symptomatic choledocholithiasis in pregnancy. Am J Gastroenterol 1994;89:1601-1602.

4. Stavropoulos S, Larghi A, Verna E, Stevens P. Therapeutic endoscopic retrograde cholangiopancreatography without fluoroscopy in four critically ill patients using wire-guided intraductal ultrasound. Endoscopy 2005;37:389-392.

5. Draganov P, Cotton PB. Iodinated contrast sensitivity in ERCP. Am J Gastroenterol 2000;95:1398-1401.

6. Chak A, Isenberg G, Kobayashi K, Wong RC, Sivak MV Jr. Prospective evaluation of an over-the-wire catheter US probe. Gastrointest Endosc 2000;51:202-205.

7. Tamada K, Inui K, Menzel J. Intraductal ultrasonography of the bile duct system. Endoscopy 2001;33:878-885.

8. Menzel J, Domschke W. Gastrointestinal miniprobe sonography: the current status. Am J Gastroenterol 2000;95:605-616.

9. Cotton PB, Lehman G, Vennes J, et al. Endoscopic sphincterotomy complications and their management: an attempt at consensus. Gastrointest Endosc 1991;37:383-393.

10. Catanzaro A, Pfau P, Isenberg GA, Wong RC, Sivak MV Jr, Chak A. Clinical utility of intraductal US for evaluation of choledocholithiasis. Gastrointest Endosc 2003;57:648-652.

11. Stavropoulos S, Larghi A, Verna E, Battezzati P, Stevens P. Intraductal ultrasound for the evaluation of patients with biliary strictures and no abdominal mass on computed tomography. Endoscopy 2005;37:715-721.

12. Jorgensen JE, Rubenstein JH, Goodsitt MM, Elta GH. Radiation 
doses to ERCP patients are significantly lower with experienced endoscopists. Gastrointest Endosc 2010;72:58-65.

13. Tsapaki V, Paraskeva KD, Mathou N, et al. Patient and endoscopist radiation doses during ERCP procedures. Radiat Prot Dosimetry 2011;147:111-113.

14. Wang HP, Huang SP, Sun MS, et al. Urgent endoscopic nasobiliary drainage without fluoroscopic guidance: a useful treatment for critically ill patients with biliary obstruction. Gastrointest Endosc 2000;52:741-744.

15. Götzberger M, Pichler M, Gülberg V. Contrast-enhanced US-guided ERCP for treatment of common bile duct stones in pregnancy. Gastrointest Endosc 2012;76:1069-1070.

16. Artifon EL, Kumar A, Eloubeidi MA, et al. Prospective randomized trial of EUS versus ERCP-guided common bile duct stone removal: an interim report (with video). Gastrointest Endosc 2009;69:238243.

17. Pohl J, Ell C. Direct transnasal cholangioscopy with ultraslim endoscopes: a one-step intraductal balloon-guided approach. Gastrointest Endosc 2011;74:309-316.

18. Weigt J, Kandulski A, Malfertheiner P. Direct peroral cholangioscopy using ultraslim gastroscopes: high technical performance with important diagnostic yield. Gastrointest Endosc 2014;79:173-
177.

19. Itoi T, Moon JH, Waxman I. Current status of direct peroral cholangioscopy. Dig Endosc 2011;23 Suppl 1:154-157.

20. Ascher SM, Evans SR, Goldberg JA, et al. Intraoperative bile duct sonography during laparoscopic cholecystectomy: experience with a 12.5-MHz catheter-based US probe. Radiology 1992;185:493496

21. Ang TL, Teo EK, Fock KM, Lyn Tan JY. Are there roles for intraductal US and saline solution irrigation in ensuring complete clearance of common bile duct stones? Gastrointest Endosc 2009;69:1276-1281.

22. Menzel J, Poremba C, Dietl KH, Domschke W. Preoperative diagnosis of bile duct strictures: comparison of intraductal ultrasonography with conventional endosonography. Scand J Gastroenterol 2000;35:77-82.

23. Jha R, Al-Kawas FH. How good is IDUS in patients with isolated biliary strictures? Am J Gastroenterol 2004;99:1690-1691.

24. Tse F, Yuan Y, Moayyedi P, Leontiadis GI. Guidewire-assisted cannulation of the common bile duct for the prevention of postendoscopic retrograde cholangiopancreatography (ERCP) pancreatitis. Cochrane Database Syst Rev 2012;12:CD009662. 